\title{
Techniques for Turbulence Tripping of Boundary Layers in RANS Simulations
}

\author{
Narges Tabatabaei ${ }^{1,2}$ (I) $\cdot$ Ricardo Vinuesa ${ }^{1,2}$ (D) $\cdot$ Ramis Örlü ${ }^{1,2}$ (D) Philipp Schlatter ${ }^{1,2}$ (D)
}

Received: 29 December 2020 / Accepted: 26 August 2021 / Published online: 24 September 2021

(c) The Author(s) 2021, corrected publication 2021

\begin{abstract}
The exact placement of the laminar-turbulent transition has a significant effect on relevant characteristics of the boundary layer and aerodynamics, such as drag, heat transfer and flow separation on e.g. wings and turbine blades. Tripping, which fixes the transition position, has been a valuable aid to wind-tunnel testing during the past 70 years, because it makes the transition independent of the local condition of the free-stream. Tripping helps to obey flow similarity for scaled models and serves as a passive control mechanism. Fundamental fluid-mechanics studies and many engineering developments are based on tripped cases. Therefore, it is essential for computational fluid dynamics (CFD) simulations to replicate the same forced transition, in spite of the advanced improvements in transition modelling. In the last decade, both direct numerical simulation (DNS) and large-eddy simulations (LES) include tripping methods in an effort to avoid the need for modeling the complex mechanisms associated with the natural transition process, which we would like to bring over to Reynolds-averaged Navier-Stokes (RANS) turbulence models. This paper investigates the implementation and performance of such a technique in RANS and specifically in the $k-\omega$ SST model. This study assesses RANS tripping with three alternatives: First, a recent approach of turbulence generation, denoted as turbulence-injection method $(k \mathrm{I})$, is evaluated and investigated through different test cases; second, a predefined transition point is used in a traditional transition model (denoted as IM method); and third a novel formulation combining the two previous methods is proposed, denoted $\gamma-k \mathrm{I}$. The model is compared with DNS, LES and experimental data in a variety of test cases ranging from a turbulent boundary layer on a flat plate to the three-dimensional (3D) flow over a wing section. The desired tripping is achieved at the target location and the simulation results compare very well with the reference results. With the application of the novel model, the challenging transition region can be excluded from a simulation, and consequently more reliable results can be provided.
\end{abstract}

Keywords Tripping · Boundary layer · CFD RANS · Laminar-turbulent transition

Narges Tabatabaei

nargest@mech.kth.se

SimEx/FLOW, Engineering Mechanics, KTH Royal Institute of Technology, Stockholm, Sweden

2 Swedish e-Science Research Centre (SeRC), Stockholm, Sweden 


\section{Introduction}

The action of forcing transition from a laminar to a turbulent boundary layer (BL) is common in wind-tunnel (WT) testing to eliminate the later transition caused by testing at reduced Reynolds numbers ( $R e$ ) (Mabey 1991). For example, at low Re and for an airfoil at stall-angle, it is essential to ensure that the transition occurs before the laminar flow separation. Boundary layer trips are traditionally also used in scale-model testing to aid in scaling the flow characteristics, where duplicating full-scale Reynolds numbers is not feasible in the WT which leads to the fact that the developed BLs on WT models do not correspond to the BLs which develop on full-scale vehicles. For this purpose, tripping devices are placed on models to hasten the BL transition from laminar to turbulent flow. In this way, the characteristics which are sensitive to the condition of the BL are more accurately simulated in tripped cases (Peterson 1969), since the transition is modeled independent of the local condition of the free-stream which may differ from case to case. Furthermore, the key idea of passive techniques is to trip the BL to re-energize the flow so that the flow remains attached (Sreejith and Sathyabhama 2018).

The skin friction, and consequently the drag, changes due to the shift of the transition position from one test to another, which defines the turbulent portion of the model. The different transition onsets in adverse-pressure-gradient (APG) flows can also result in larger separation regions farther downstream, with the corresponding impact on aerodynamic performance (Tabatabaei 2018). Similarly, laminar-turbulent transition is known to affect the separation location and consequently lift and drag may be substantially affected. In addition, it is sometimes possible to duplicate the relative thickness of the full-scale turbulent BL at certain locations on the WT model by fixing transition at the proper location (Blackwell 1969).

Most studies focusing on the physics of turbulent BLs employ tripping to promote early and robust transition to turbulence (Erm and Joubert 1991, Marusic et al. 2015, Sanmiguel Vila et al. 2017). For instance, different tripping devices were studied, in the context of a flat-plate BL (Head and Bandyopadhyay 1981, Rengasamy and Mandal 2017). Roughness elements were also used to force transition in order to eliminate the transitional effects (Iverson et al. 2019; Reshotko 2001). The wide and continuous application of tripping in WTs (dos Santos et al. 2020) motivated the use also in numerical methods to model similar effects in order to replicate the experimental data. Therefore, tripping methods evolved beside the numerical transition models that attempted to model the natural transition process. Referring to the variety of transition types and the complexity of modeling the complex physical interactions and mechanisms leading to transition, tripping models have the advantage of simplicity, which results in a much lower uncertainty. For instance,, various tripping strategies were assessed over a flat plate by direct numerical simulation (DNS) (Schlatter and Örlü 2012) and were later implemented in large-eddy simulation (LES) for a 2D airfoil (Lewis 2017). However, these high-fidelity methods cannot simulate the complete model of WT sections due to the high computational costs. For six-equation RANS models, i.e. Reynolds-stress Model (RSM), a tripping methodology was implemented and compared with experimental data for fixed transition of airfoil flows (Gerolymos and Vallet 2013). Among eddy-viscosity RANS models (EVM), the one-equation turbulence model of Spalart-Allmaras (SA) contains a trip term from the beginning in 1992 (Spalart 1992). These authors used the word trip "to mean that the transition point is imposed by an actual trip, or natural but obtained from a separate method" (Spalart 1992). The trip version of the SA model, named as SA-Ia, is rarely used, because the model is 
most often employed for fully-turbulent (FT) applications (Rumsey 2021). Its trip term was found to be inadequate to force transition at a specified location, specifically for hypersonic flows (Roy and Blottner 2001).

The $k-\omega$ SST model, as the most common two-equations EVM model, was developed in 1994 (Menter 1994). The formulations of both turbulence transport equations ( $k$ and $\omega)$ are based on the features of FT-BL and so the initial laminar region, and consequently the transition part, are not modeled accurately. Such a formulation induces an early turbulent viscosity buildup (as if for e.g. there is a surface roughness) and therefore causes FT flow over the region which is laminar in the physical model and leads to over-estimating the drag (Tabatabaei 2018; Acarer 2020). RANS-based BL transition algorithms have been broadly considered in literature since few decades ago (Walters and Cokljat 2008; Gokdepe 2015). Most commonly transition models consist of two main parts: 1 . Define the laminar, transition and FT regions, i.e. the intermittency $(\gamma)$ distribution. This can be done via two, one, or even zero transport equations (Langtry 2006; Menter et al. 2015; ANSYS 2015); 2. Apply the modifications into the turbulence model, i.e. in the $k$ and $\omega$ equations, which is referred to as 'coupling with $k-\omega$ SST' model. The currently available transition models in RANS are typically based on empirical correlations, but are not specifically aimed at representing the physical mechanisms in the transition process. As discussed by Langtry (Langtry 2006), "They do not attempt to model the physics of the transition process (unlike e.g. turbulence models), but form a framework for the implementation of transition correlations into general purpose CFD methods". They are basically designed to cover the standard 'bypass transition', as well as flows in low free-stream turbulence environments (since the transition location is correlated with the free-stream turbulence intensity, based on laboratory data) (ANSYS 2015). In addition to becoming unstable (in terms of convergence) (Acarer 2020), it was observed that setting a lower value for the free-stream turbulence in the CFD simulations would result in a later transition prediction than observed in the physical model (Luckring et al. 2016). Apart from the pros and cons of such approaches in simulating the transition process, recent studies show that "There is potential for uncertainty or error in simulating the forced transition case with RANS models"(Acarer 2020). In order to initiate transition at the same location as the experimental data, zigzag tapes were used, but the uncertainties inevitably appeared even in the calculations of the integrated parameters, e.g. the total power of the whole turbine rotor. Certainly, it is more challenging when a point-to-point comparison is intended, e.g. in chordwise $C_{p}$ distribution. Similarly, turbulence tripping was implemented in RANS using a specific type of obstacle in the geometry, which caused flow disturbances that facilitated the transition from laminar to turbulent flow (Sreejith and Sathyabhama 2018). Although a sudden jump in the local pressure was achieved, a spurious small vortex emerged downstream of the obstacle.

With the target of replicating the same transition point as in the experiments, and remove the uncertainty in the numerical model of forced transition, this study investigates several numerical approaches that mimic the effect of a turbulence trip. Tripping in experiments is not always implemented at the natural transition point, rather the target point of forced transition may shift for different reasons such as control. Therefore, a flexible numerical approach is required to control the flow condition, as it has already been investigated in LES and DNS. The present work considers different methods to implement tripping with RANS as well as the assessment of each of them compared to WT experiments, beside the similar tripping approaches in LES and DNS. The ultimate goal of the project is to develop a numerical simulation approach which can represent the complex experimental setup and measurements in a WT, reduce the uncertainty in design of a setup, and thus increase the 
fidelity of a campaign. This motivates to include three-dimensional (3D) setups as a part of this research, such as complete WT setups, in the framework of a 'virtual WT'.

This article is structured as follows: First, the numerical setup is described in Sect. 2, which includes a brief description of three different test cases to assess the different approaches, as well as the specification of the numerical simulation (Sects. 2.1 and 2.2 respectively). The fundamentals and background of the injection method is explained in Sect. 3. The method is investigated for the three test cases and the results for each case are assessed through the comparison with higher fidelity data from LES or experiments. Furthermore, tripping methods in DNS are compared with the RANS in Sect. 3.1.1, where the flat plate is discussed. An alternative method of tripping is assessed in Sect. 4, including the method fundamentals together with some sample results. Finally, in Sect. 5, we propose a formulated method of implementation for that idea, which is inspired from the methodology of Sect. 4. Conclusions are drawn in Sect. 6 together with recommendations for future directions.

\section{Numerical Setup and Test Cases}

In this section, the computational framework is introduced together with a description of the three flow cases studies.

\subsection{Test Cases}

To assess the performance of the tripping methods in RANS, we study three different cases, representative of a broader range of application cases. These range from a canonical BL in a statistically two-dimensional setting to a complicated fully three-dimensional WT setup, where experimental data serve as reference data. Here, we list the cases briefly, and defer a more detailed discussion to where the results are presented. The test cases documented in the paper correspond to the cases where the investigated tripping methods are most appropriate, i.e. in situations where natural transition needs to be avoided or the tripping location needs to be fixed, and where trips are used in experiments. We limit ourselves to subsonic flow, as most of the reference data (from experiments and computations) is available in this regime. However, an extension to higher speeds is certainly possible, keeping in mind that even for experiments the effect of tripping devices on supersonic turbulent boundary layers is much less studied (Bross et al. 2018).

- Test case 1: Flat plate turbulent BL with zero pressure gradient (ZPG).

The flow over a flat plate is modeled as a two-dimensional (2D) domain including the streamwise direction, as well as the wall-normal direction. Zero static pressure is applied at the surrounding top boundary of the domain. The $R e_{L}$ is $3.4 \times 10^{6}$, based on the plate length $L$.

- Test case 2: Two-dimensional airfoil.

The NACA4412 profile is the reference airfoil selected for this study, as in similar studies (Coles and Wadcock 1979). That is investigated both in free-flight condition and inside a WT. A large circular domain is selected to represent the corresponding farfield as in the free-flight condition. The diameter of the domain is 50 times of the airfoil chord length $(c)$ and the airfoil is located at the centre of the domain. The same airfoil is simulated in a confined computational domain replicating the physical experiment in 
a WT. The WT is modelled according to a concurrent experiment (Mallor et al. 2019), performed in the Minimum-Turbulence-Level (MTL) WT (Österlund 1999; Lindgren and Johansson 2002; Sanmiguel Vila et al. 2017) at KTH Royal Institute of Technology. The mid-height section of the tunnel is considered as an one-layer domain in a 2D simulation. The maximum Mach number for the implemented experiments is $\approx 0.2$ in the tunnel. The Reynolds number based on freestream velocity and the chord length $C$ is $R e_{c}=4 \times 10^{5}$ and the angle of attack ranges from 5 to $11^{\circ}$.

- Test case 3: Three-dimensional wing in a WT.

The 3D computational domain is constructed according to MTL WT, however considering now also the full side-walls, ceiling and floor of the tunnel to make this test case fully three dimensional. The test section is $7 \mathrm{~m}$ long and it has a cross-section area of $1.2 \times 0.8 m^{2}$. An illustration of the WT test section is shown in Fig. 1. The same $R e_{c}$ and angles of attack as in the $2 \mathrm{D}$ cases were considered.

\subsection{Computational Methods}

For each considered computational case, a multi-block structured hexahedral mesh is generated. ANSYS ICEM CFD (ANSYS 2011) is used to generate the more complicated meshes used for the WT geometry, both in $2 \mathrm{D}$ or $3 \mathrm{D}$, and the flat plate boundary-layer cases, while Gmsh (Geuzaine and Remacle 2009) was used for the meshes pertaining to free-flight airfoil setup with the corresponding large far-field. Two-dimensional simulations include a single cell layer. The simulations are based on a finite volume discretization via the open-source OpenFOAM (OF) code with the SIMPLE (semi-implicit method for pressure linked equations) and PIMPLE (merged PISO-SIMPLE) algorithms, where PISO stands for pressure-implicit with splitting of operators (Greenshields 2019). Open-source code OF is mainly used in this research to implement the various techniques, both through built-in turbulence models and new developed libraries. In addition, A hybrid finiteelement/finite-volume approach to discretize the RANS equations is also used as implemented in the commercial CFD code ANSYS CFX (ANSYS 2015) for the assessments in Sect. 4. All the other analysis are implemented using OF. As the base turbulence model,

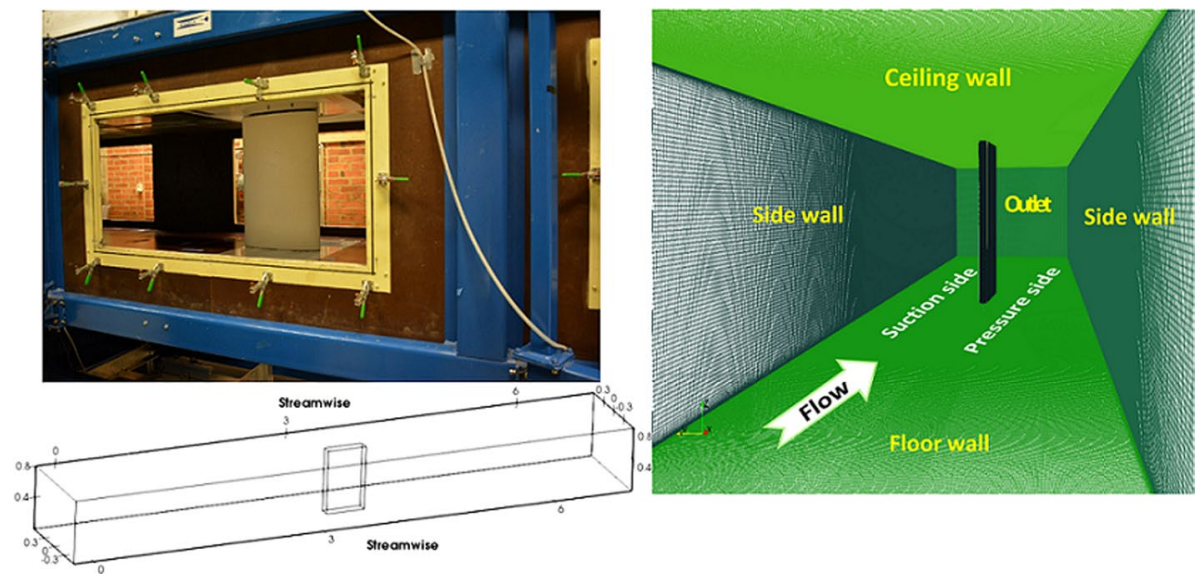

Fig. 1 Illustration of the wing located in WT test section; left: MTL test section and schematic of the test section scales, right: the CAD model of the 3D domain 
a two-equation EVM is considered: $k-\omega$ SST (Menter 1994). The first grid point in the wall-normal direction is always located at a distance from the wall below one viscous unit (i.e., in terms of the viscous length scale $\ell_{*}=v / u_{\tau}$, where $v$ and $u_{\tau}$ are the fluid kinematic viscosity and friction velocity, respectively). Appropriate wall functions are set over the walls for different properties. Zero-gradient boundary conditions are applied for the outflow, while the inflow conditions are defined with fixed values according to the realistic turbulence intensity $(\mathrm{Tu})$ for a high-quality wind tunnel $(\mathrm{Tu}=0.1 \%)$. It is important to keep the free-stream as clean as possible in order to establish a canonical BL. Consequently, the eddy-viscosity ratio at the inflow is approximately 0.33 for the MTL models. The validity of the simulation setups have been investigated in terms of the computational mesh efficiency, and the agreement of the results with the reference data as shown in Tabatabaei et al. (2021) and e.g. Sects. 3.2 and 3.3.

\section{Injection of Turbulent Kinetic Energy ( $k l)$}

We start with describing a turbulence-generation mechanism, which was recently adopted by Fahland (2019) for the RANS simulation of the flow around an airfoil. This method marks the starting point of our analysis. We denote this as 'injection method' $(k \mathrm{I})$, and it is based on directly modifying the turbulent kinetic energy, $k$ at target trip point. Note that the injection of extra $k$ effectively promotes transition at the position or shortly downstream of it. The modelled transport equation for $k$ is may be written as

$$
\frac{\partial(\rho k)}{\partial t}+\frac{\partial\left(\rho u_{j} k\right)}{\partial x_{j}}=P_{k}-D_{k}+\frac{\partial\left[\left(\mu+\sigma_{k} \mu_{t}\right) \frac{\partial k}{\partial x_{j}}\right]}{\partial x_{j}}+S_{k},
$$

where $P_{k}$ and $D_{k}$ denote the production and dissipation terms respectively (Menter 1994). The coefficients $\mu$ and $\mu_{t}$ denote the dynamic viscosity and turbulent dynamic viscosity, respectively and the corresponding term (the 3rd term on the right-hand side) refers to the diffusion of $k$. The last term on the right-hand side, $S_{k}$ is included to account for the sources of turbulent kinetic energy. The source term can be defined in different ways, such as a semi-implicit term including explicit and implicit contributions (Greenshields 2019). The $k \mathrm{I}$ approach is based on adding a local $S_{k}$ at the target trip point so that the flow becomes FT immediately, in the same way as in the experimental tripping. In $k$ I tripping $S_{k}$ is introduced as an explicit parameter. Furthermore, the standard $k-\omega$ SST model leads to an early transition so that the BL becomes FT even before the physical transition position. The redundant turbulence generation in the standard $k-\omega$ SST and the consequent early transition, will be discussed in Sect. 4. In order to ensure the turbulence model does not lead to a premature deviation from the laminar solution, the value of $k$ can be set to zero in the domain just upstream the tripping. This constraint is set to avoid an over-estimation of $k$ in the laminar region, which is anyway calculated from the FT equations in the $k-\omega \mathrm{SST}$ model.

There are two main parameters in this method: the injection 'area' and its 'magnitude' (Fahland 2019). Note that $S_{k}$ value, as the injection magnitude, should be large enough to raise the local skin-friction coefficient $C_{f}$ at the tripping point. These two parameters should be tuned in terms of the available reference data for comparable cases. An advantage of the $k \mathrm{I}$ method is the simplicity of the implementation, because the source terms can typically be externally modified without changing the core of the solver. 
Figure 2 illustrates how the injection area is specified for a flat plate and a wing model, similar to tripping in the experiments. The laminar region, i.e. the $k=0$ area, is defined to implement the turbulence constraint, which was expressed in the beginning of this section. This is henceforth denoted 'forced laminar' region throughout the remainder of the paper. The approximated depth of the injected area is suggested to be of the momentum thickness $\theta$ size at the tripping location (Fahland 2019), since the tripping should be located inside the $\mathrm{BL}$ region. The $k_{1}$ part in Fig. 2-right shows the injection section, as well as the injection bar assigned on the wing in Fig. 2-left.

The following sections assess the idea of local turbulence source as a tripping method in RANS. The presented results are obtained through the $k I$ method and via an iterative process for the test cases described in Sect. 2.1.

\subsection{Test Case 1: Flat Plate}

The ZPG flow over a flat plate is tripped with the application of the $k$ I method. The results are shown in terms of $C_{f}$, which is the normalized wall shear stress at the wall.

\subsubsection{Comparing with Tripping Parameters in DNS}

The specific numerical model of tripping was first introduced in DNS for a ZPG flow. To evaluate the $k$ I tripping in RANS, in this part we focus on the low- $R e_{\theta}$ trends, which were studied in Schlatter and Örlü (2012) via the use of different tripping parameters in DNS. The typical reference TBL data, as referred to also in Sect. 3.1.2, are mainly valid for higher $R e_{\theta}$. They were found to be ambiguous at lower $R e_{\theta}$, indicating that the BL development at these low $R e$ are strongly based on the way turbulence was generated e.g. tripping mechanism. From the tripping scenarios considered in Schlatter and Örlü (2012), we consider the following three generic cases to compare with RANS: low-amplitude (LA), low frequency (LF) and high-frequency (HF) tripping methods. These cases are plotted in Fig. 3, where the trip is applied at $R e_{\theta}=180$. For the RANS, the standard $k-\omega$ SST model with no explicit transition/tripping model is included. The $k \mathrm{I}$ method results are the same ones in Fig. 4b.
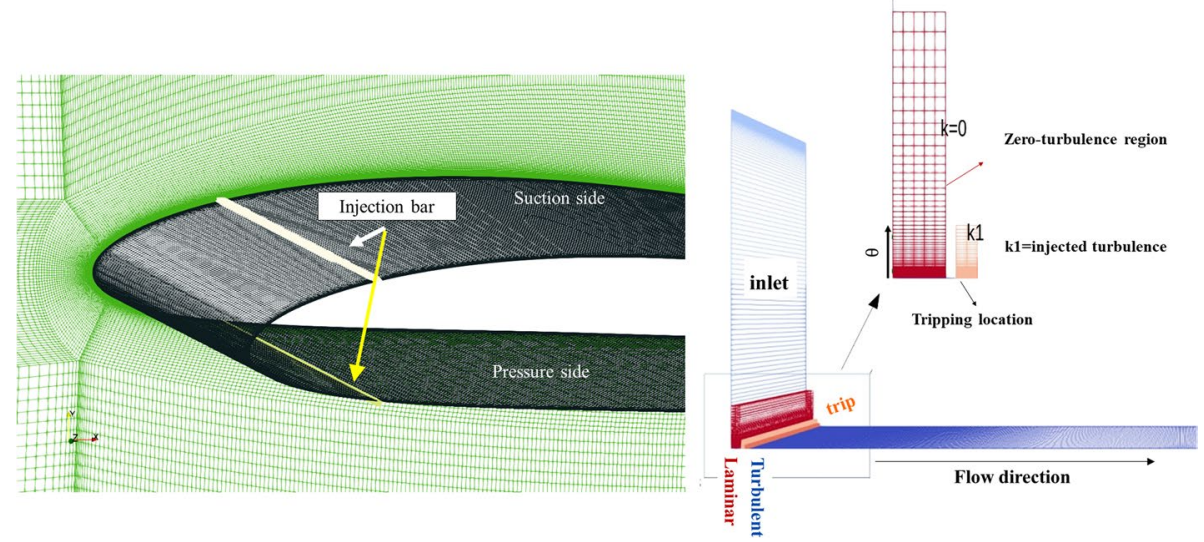

Fig. 2 Illustration of the injection tripping procedure in various flows relevant for the present work. See text for more details 


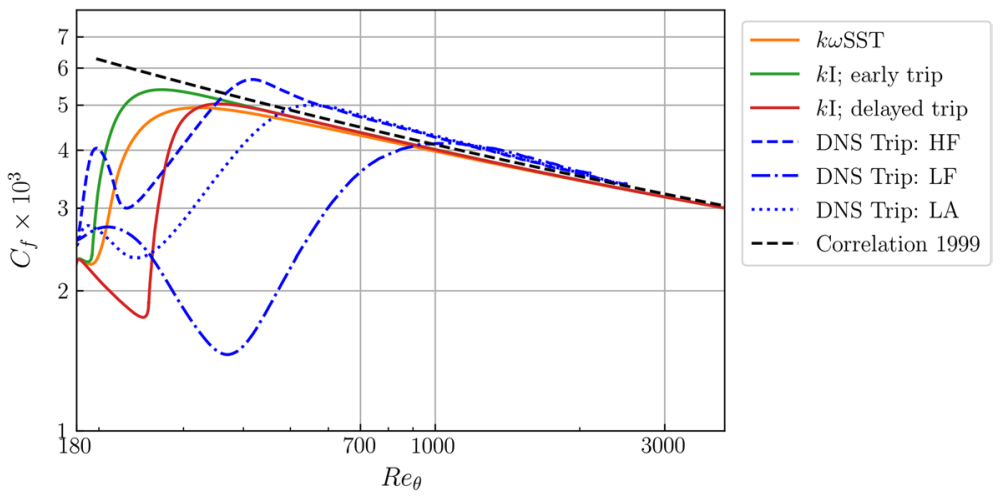

Fig. 3 Comparison of the injection methods with various tripping parameters implemented in DNS (Schlatter and Örlü 2012). FT baseline is according to Österlund (1999) (Correlation 1999)

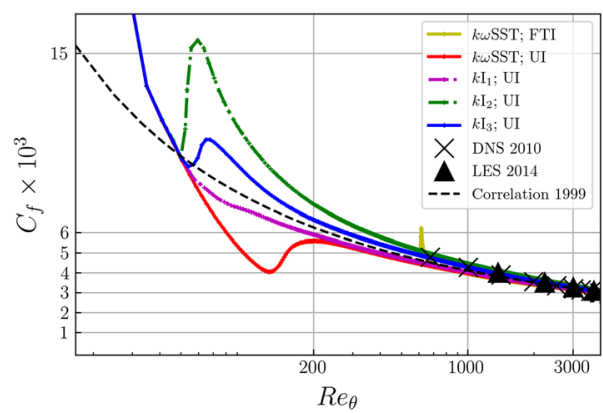

(a)

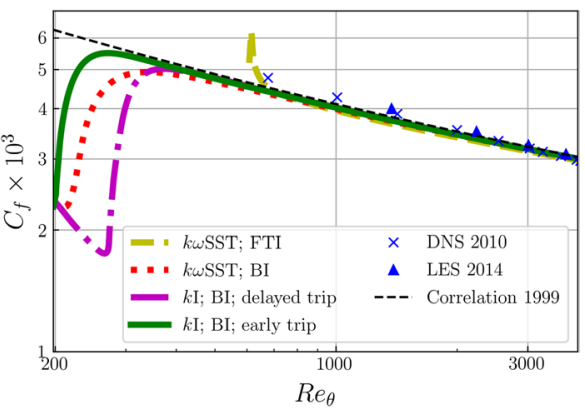

(b)

Fig. 4 Injection tripping for ZPG flat plate; a uniform and b Blasius inflow profiles ('UI' and 'BI' respectively); FT baselines are based on Schlatter and Örlü (2010), Eitel-Amor et al. (2014), Monkewitz et al. (2007), and Österlund (1999) for DNS 2010, LES 2014, Correlation 2007, Correlation 1999, respectively; 'FTI' denotes the FT profile at inflow from DNS; $R e_{\theta}$ and $C_{f}$ are in $\log$ scale

For the DNS a different behaviour was observed in the region $R e_{\theta}=180-1000$, which was due to the transition mechanisms. Although $C_{f}$ increases immediately at the tripping $\left(R e_{\theta}=180\right)$, it does not reach the FT baseline directly, with a distinct behavior, as discussed by Schlatter and Örlü (2012). However, all methods come together at a certain $R e_{\theta} \approx 1000$ within the FT region. In the $k$ I method, the jump in wall shear occurs right at the target tripping location and the curve rapidly adapts to the FT baseline without significant overshoot or undershoot and a comparably short development length. From the data shown in this section, we can conclude that injection of kinetic energy into the BL provides an effective way to trip a BL, since it shows similar results as in DNS tripping. The resulting turbulent flow quickly adapts to the canonical form of the turbulent BL, with shorter development length as non-optimal tripping as studied by DNS. 


\subsubsection{Parameter Investigations}

Two different states were considered for the inflow boundary condition: uniform (UI) and Blasius (BI) profiles in Figs. 4a and Figs. 4b respectively. Different reference cases are considered as being representative of a turbulent BL. The ZPG DNS results under consideration are reported in Schlatter et al. (2009) and Schlatter and Örlü (2010). Similar results based on LES are used as a baseline, and they are documented in Eitel-Amor et al. (2014). Furthermore, we also show empirical correlations for $C_{f}$, considering the correlations by Monkewitz et al. (2007) and Österlund (1999). The standard RANS ( $k-\omega$ SST) is included in the plots with DNS data as inflow profile (denoted as 'FTI'), as well as the UI inflow. This means that the turbulence model is based on the assumption of FT flow everywhere. As in the case of the DNS and LES baselines, we do not include data for $R_{\theta}<600$ because the flow should develop over a sufficient length to reach the FT condition. The corresponding yellow line shows the onset of the FTI simulation, which exhibits a trend that merges with the other FT curves. The injection point for the uniform-inflow cases in Fig. $4 \mathrm{a}$ is located at $R e_{\theta} \approx 52$ which corresponds to a typical position in a WT and previous tripping simulation (Schlatter and Örlü 2012). In Fig. 4b, two scenarios are tested to assess the method efficiency: early and delayed tripping. First the flow is tripped immediately after the domain inlet as an 'early-trip'. Conversely, in the case denoted as 'delayed trip', the simulation keeps the flow laminar for some distance before it is tripped. The injection points for these two scenarios are located at $\Delta R e_{\theta} \approx 40$ and 95 respectively, where $\Delta$ is reported with respect to the inflow $\left(R e_{\theta} \approx 175\right)$. The results show that the $k$ I method can efficiently control the flow state, while the BL development is also preserved. Since $k \mathrm{I}$ does not provide definite values for the $k$ injection magnitude and area, a sensitivity analysis is performed and the results are shown for three arbitrary configurations of the injection 'area' and 'magnitudes' with subscripts $1-3$. For the $k I_{1}$ method, a lower magnitude of the injection is considered and compared to $k I_{2}$, where as the injection magnitude at $k I_{2}$ is 20 times of the one at $k I_{1}$. In $k I_{3}$ a combined condition is used by splitting the injection area into two parts. Then the injection magnitudes in the two injection regions are 2 and 4 times of $k I_{1}$ case, respectively.

The logarithmic plots in Fig. 4 show that all the $k$ I methods adapt to the baseline within a short length $\left(\Delta R e_{\theta}=200\right)$ and the resulting FT flow matches with the FT reference data. In spite of the slight differences in Fig. 4a, the method functions well for all three cases and the intended tripping is achieved for all cases despite the variations of the method's parameters.

As a general feature of $k \mathrm{I}$ for all cases, an immediate response is indicated by a $C_{f}$ -increase at the target trip location, in addition to the controllable transition point. Table 1 summarizes the specifications of tripping for the above cases. The transition onset is considered to be the location at which $C_{f}$ starts to increase after the laminar region with decreasing $C_{f}$. The injection location is reported as the target trip point, and fully-turbulent (FT) flow is assumed to be at the location where $C_{f}$ has reached its maximum value within the transition/development length (denoted as $\Delta R e_{x}$ ). With the $k \mathrm{I}$ approach, transition starts immediately at the target trip point and the development length is quite short, while the standard $k-\omega$ SST needs a longer distance to reach a fully turbulent condition. The transition onset with the standard $k-\omega$ SST is upstream of the natural transition location and it is not controllable and thus a target trip point cannot be defined.

In all the cases in this section, as well as the following ones, the free-stream turbulence $(T u)$ was selected to be low in order to assess the most relevant scenario for 
Table 1 Spatial distribution of the tripping over the flat plate. All the positions on the plate are expressed as $R e_{x} \times 10^{-4}$ and $\Delta R e_{x}$ denotes the development length

\begin{tabular}{lllllll}
\hline Inflow & Model/Scenario & Target Trip & $\begin{array}{l}\text { Transition } \\
\text { Onset }\end{array}$ & FT Location & Forced Laminar & $\Delta R e_{x}$ \\
\hline \#UI & $k-\omega$ SST & - & 2.5 & 4.2 & - & 1.7 \\
\#UI & $k I_{2}$ & 0.4 & 0.4 & 0.6 & 0.3 & 0.2 \\
\#UI & $k I_{3}$ & 0.4 & 0.4 & 0.7 & 0.3 & 0.3 \\
\#BI & $k-\omega \mathrm{SST}$ & - & 1.5 & 5.7 & 0.3 & 4.2 \\
\#BI & $k I_{\text {early }}$ & 0.4 & 0.4 & 2.6 & 6.6 & 2.2 \\
\#BI & $k I_{\text {delayed }}$ & 6.7 & 6.7 & 9.7 & & 3.0 \\
\hline
\end{tabular}

tripping. However, we investigated the effect of $T u$ within a wide range of intensities and increased it up to 30 times the base value. The result is shown in Fig. 5 for the flat-plate case used as the delayed-trip scenario in Fig. 4b. Increasing $T u$ by a factor of $10(T u=1 \%)$ led to no changes on the $C_{f}$ development. With $T u=3 \%$ the streamwise evolution of the flow field was modified irrespective of tripping. In this paper, the inflow Reynolds number $R e_{i}$ is selected according to the typical values in the wind tunnel, but the same tripping is functional at higher $R e_{i}$ without the need for an additional calibration.

\subsection{Test case 2: 2D airfoil}

The tripping models with RANS simulations were also applied to a NACA4412 wing section in free-flight conditions, and compared with a well-resolved LES of the same case (Vinuesa et al. 2018) tripped with the method in Schlatter and Örlü (2012) at $5^{\circ}$ angle of attack. Using the $k \mathrm{I}$ method discussed above, we prescribe the transition location to be the same as that in the original LES, i.e. $x / c=0.1$. The computational domain for the isolated airfoil, together with the velocity contour are shown in Fig. 6a. A detailed view of the airfoil, in addition to the $k$ distribution, are also shown. The abrupt jump in the $k$ magnitude is the consequence of tripping at the marked location. As the other result of tripping chord-wise distribution of $C_{f}$ is plotted for this isolated airfoil. Fig. $6 \mathrm{~b}$ shows that the $C_{f}$ distribution from the RANS is in excellent agreement with the reference data by Vinuesa et al. (2018).

A similar process was conducted for a NACA4412 airfoil inside MTL WT and in different angles of attack. Tripping was implemented on the airfoil suction side using $k \mathrm{I}$ approach. The $k$ I tripping coupled with $k-\omega$ SST resulted in an instantly turbulent BL which was at the same target trip point of the model wing in the wind tunnel.

\subsection{Test case 3: 3D wing}

Finally, the $k$ I tripping is analyzed in a fully three-dimensional situation. A 3D RANS simulation of the same wing, shown in Fig. 2, was performed at $11^{\circ}$ angle of attack. The same tripping location as that in the actual WT experiment, documented by Mallor (2019), is considered. The mid-height section is selected to plot the chordwise distribution of pressure coefficient $C_{p}$ for both experiments and RANS. The measured data and simulation results, with 
Fig. 5 Injection tripping with various free-stream turbulence levels, and the effect on $C_{f}$ over a flat plate up to $R e_{x} \approx 67 \times 10^{4}$

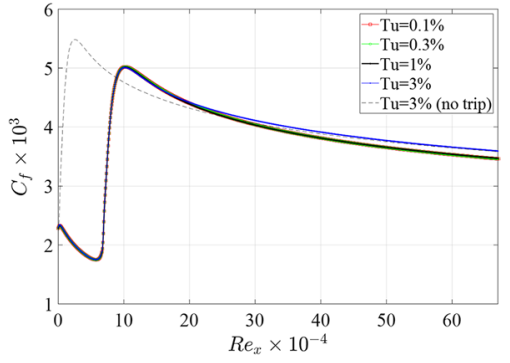

and without tripping are compared in Fig. 7. Static pressure $p$ is non-dimensionalized with the dynamic pressure $P_{d}$ :

$$
C_{p}=\left(p-p_{\infty}\right) / P_{d}
$$

It turns out that in the experiments only marginal differences between tripping and notripping could be seen; this is probably due to the fact that natural transition occurs at this comparably high angle of attacks (near stall). Conversely, the RANS clearly shows distinct profiles for the two cases. The 3D RANS with standard turbulence model results in a lower suction level compared to the experiments, while a close agreement is achieved through the proposed tripping technique. The suction peak (upstream of the trip location) at the mid-height plane is affected by $k \mathrm{I}$ method, which indicates the importance of the accurate development of the side-walls BL. Without prescribing a tripping position, the RANS development, specifically at the intersection of the wing and the ceiling, is unclear, but by setting and optimising a tripping position, we can adjust the development to match that of the experiment. The inaccuracy in the properties of the surrounding-walls BL may transmit to the mid-section and disturb the calculated $C_{p}$, while the combination of laminarization and injection specifically on the ceiling avoids this. This indicates the significance of tripping in the global flow characteristics beside its effect on the local variations. The $C_{f}$ distribution also confirms the occurrence of an instant transition at the desired tripping location (not shown here). The tripping was also used successfully for the lower angles of attacks, i.e. $\mathrm{AOA}=5^{\circ}$ and $8^{\circ}$ (not shown). These results demonstrate the importance of properly fixing the transition location for both experiments and simulations, in particular if comparison is the goal of a specific study.

\section{Intermittency models as a tripping tools (IM)}

The so-called $\omega$-based RANS models (Menter 1994) were developed based on the FTBL properties, so that $P_{k}$ and $D_{k}$ are overestimated in equation (1) in non-turbulent regions. The most common transition models, known as intermittency models, are according to the local correlation-based transition modelling (LCTM) concept so that experimental correlations are integrated into standard convection-diffusion transport equations using local variables. They enter into the $k$ and $\omega$ equations via the intermittency term $\gamma$, where the equations revert to the original $k-\omega$ SST model for $\gamma=1$. The main difference between the various transition models is in how they define the $\gamma$ distribution. For instance, Langtry (2006) developed the $\gamma-R e_{\theta}$ model with two transport equations. By removing some deficiencies of the $\gamma-R e_{\theta}$ model, Menter et al. (2015) introduced a one-equation model in 2015. Furthermore, ANSYS provides 
Fig. 6 Injection tripping $(k \mathrm{I})$ for an airfoil in a 2D setup. (a) Airfoil in a free-flight configuration, view of the complete computational domain and the $k$ contours. (b) Chordwise distribution of Skin-friction coefficient, $C_{f}$ over the airfoil suction side: LES Vinuesa et al. (2018) and RANS (denoted as $k \mathrm{I}$ ), both tripped at $x / c=10 \%$

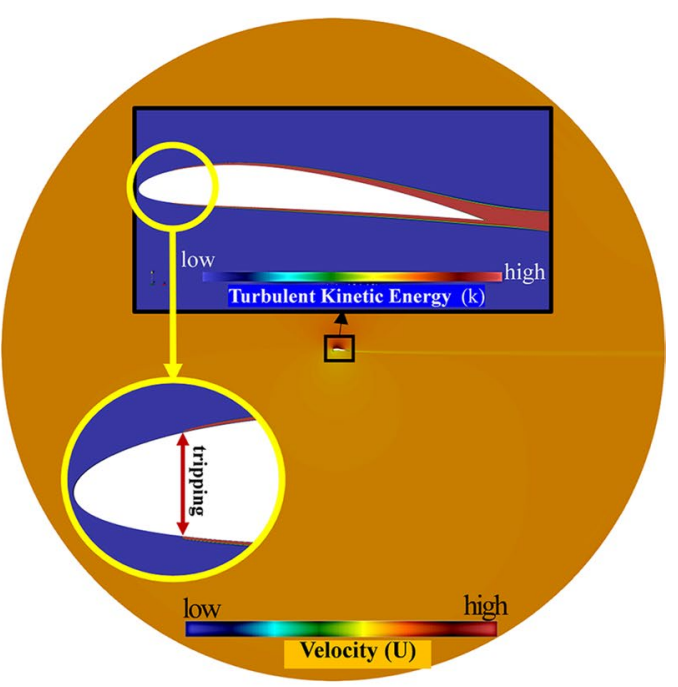

(a)

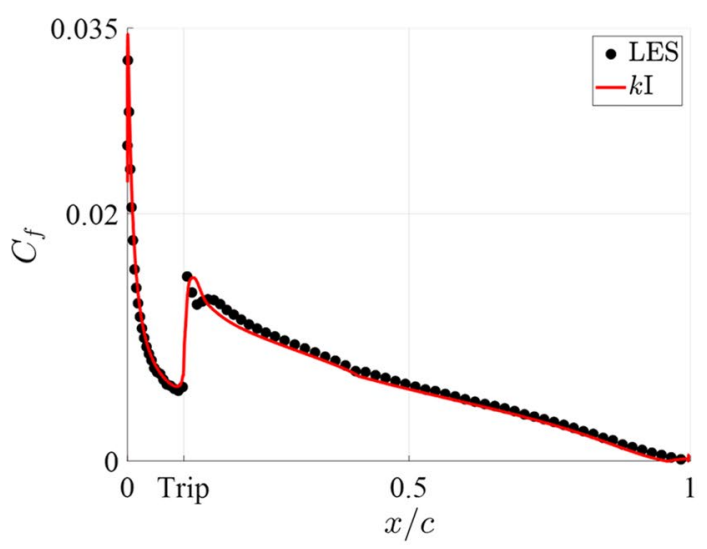

(b)
Fig. 7 Pressure-coefficient distributions at mid-height section, for tripped and untripped RANS, compared with experimental results (Mallor 2019) for the 3D wing model in the WT

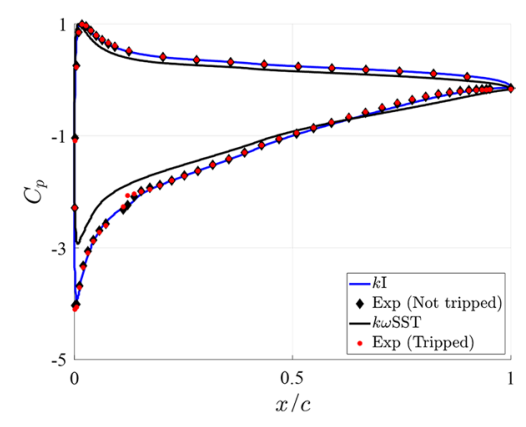


the option of a zero-equation model, in which $\gamma$ can be defined as an a-priori known function, instead of being calculated through a transport equation (ANSYS 2015). Any of the above transition models (with zero, one or two extra transport equations) can be coupled with the $k$ and $\omega$ equations. Independent of the method of defining the $\gamma$ distribution, the corresponding terms in the $k$ and $\omega$ transport equations are modified. It should be noted that the modifications, due to $\gamma<1$, are limited to the $\mathrm{BL}$ and the free-stream flow is unchanged by setting $\gamma=1$ further away from the wall.

When using tripping to render the flow turbulent, the $\gamma$ distribution is known, but instead of varying from 0 to 1 within a length, a rather sharp jump from 0 to 1 is intended. That describes the common point of the zero-equation intermittency model and the tripping: $\gamma$ is predefined in both. The assessment in this section is to discuss whether the immediate forced transition would be achieved with the application of the existing intermittency formulations. We call these methods intermittency method $I M$ in this paper. It should be noted that we are not investigating the fidelity of these methods to model the complete transition process, but the usability of these methods for tripping.

Mathematically, the main modifications due to the intermittency relate to the $k$ equation in the turbulence model as follows:

$$
\begin{gathered}
\frac{\partial(\rho k)}{\partial t}+\frac{\partial\left(\rho u_{j} k\right)}{\partial x_{j}}=\tilde{P}_{k}+P_{k}^{\lim }-\tilde{D}_{k}+\frac{\partial\left[\left(\mu+\sigma_{k} \mu_{t}\right) \frac{\partial k}{\partial x_{j}}\right]}{\partial x_{j}}+S_{k} \\
\tilde{P}_{k}=\gamma P_{k} \\
\tilde{D}_{k}=\max (\gamma, 0.1) \cdot D_{k} .
\end{gathered}
$$

Equation (2) is the most recent formulation of the intermittency model (Menter et al. 2015) and it has three differences with respect to the original formulation of the $k-\omega$ SST model (see equation (1) from Menter (1994). By replacing the normal production term $P_{k}$ with $\tilde{P}_{k}$, the turbulence production becomes zero in the laminar regions, where $\gamma$ is zero, and it increases to the normal production magnitude as the flow becomes FT $(\gamma=1)$. Similar ideas are considered for the turbulence destruction $\tilde{D}_{k}$.

Considering the intermittency value as a measure for the progress of transition, it is possible to modify the blending function of $\omega$-equation (not shown here). According to the principle of the $k-\omega$ SST model, the model switches between $k-\omega$ and $k-\epsilon$ near and far from the wall respectively (Menter 1994). In the center of the laminar BL, the $k-\omega$ model is appropriate, as well as in the transitional region, while this is neglected with the assumption of FTBL in the original $k-\omega \mathrm{SST}$. Therefore, the transition models correct the blending function with respect to the $\gamma$ magnitude and the $k-\omega$ model is kept operational until the flow becomes FT; details can be found in Menter et al. (2015) and Langtry (2006).

It should be noted that in the original formulation of the $\gamma-R e_{\theta}$ model, there was no $P_{k}{ }_{k}^{\lim }$ parameter, and thus the $\gamma-R e_{\theta}$ model can be considered as the reference transition model in which $P_{k}^{\lim }=0$. The term $P_{k}^{\lim }$ was introduced into the $k$-equation in the more recent models (Menter et al. 2015) to overcome the 'long development length' necessary to produce turbulence inside the BL. To ensure the proper generation of $k$ at the transition point for very low values of free-stream turbulence, $P_{k}{ }_{k}$ plays the role of an additional production term. The final formula is shown below where $S$ and $\Omega$ are respectively the 
magnitudes of strain rate and the absolute vorticity rate. Note that $R e_{v}$ is the local vorticity Reynolds number, defined as

$$
R e_{v}=\rho y^{2} S / \mu,
$$

where $y$ is the wall distance, see the details in Menter et al. (2015). Then $P_{k}{ }^{\lim }$ may be formulated as

$$
\begin{aligned}
P_{k}^{\lim } & =5 \max (\gamma-0.2,0)(1-\gamma) \\
& \min \left[\max \left(\frac{R e_{v}}{2.2 \times 1100}-1,0\right), 3\right] \max \left(3 \mu-\mu_{t}, 0\right) S \Omega .
\end{aligned}
$$

With the purpose of tripping at a target trip point, we assessed the application of the different intermittency models. Table 2 lists results for test cases \#1 and \#2 (Sect. 2.1), in addition to the $k \mathrm{I}$ method. Two features are intended: first the short transition/development length; and second the FT flow right at the target trip location. There are different solvers in which these transition models are implemented. The $\gamma-R e_{\theta}$ model (i.e. equation (2) with $P_{k}^{\lim }=0$ ) is the basis of the built-in turbulence model 'kOmegaSSTLM' in OF (Greenshields 2019), denoted as 'OF-LM' in the table. All variations with zero, one and two equations are denoted as 'intermittency models' in ANSYS CFX. Although the zero-equation intermittency model is still referred to as a 'transition model with a predefined intermittency', we assessed the application of it, as a 'tripping' tool in order to see whether it can result in an immediate transition. By taking the tripping location as the intermittency-switch point, $\gamma$ can be defined as a geometrical spatial distribution. The $P_{k}{ }^{\lim }$ term is designed so that it is not active in laminar and turbulent regions. This term is only activated during the development length, which almost does not exist when tripping, except for the short region at target trip location $(\gamma=0.5)$ : Indeed, the transition is intended to be an abrupt change, instead of a gradual process within a certain length. To evaluate the effect of this parameter as a tripping stimulator, a small region is considered similar to the injection zone in Fig. 2, in which $\gamma$ has a value between 0 and 1. Again the whole model with Eq. (5) defining $P_{k}^{\lim }$ was implemented in OF as a turbulence model with transition model, denoted as 'OF-IM'. The 'target trip' term in Table 2 is set according to the tripping location of the reference wing in our MTL tunnel for test case \#2, and at two selected positions for 'early' and 'delayed trip' scenarios for test case \#1. The mentioned range for $R e_{x}$ for target trip shows the scale of the small injection area (if included), corresponding to the area with $\gamma=0.5$ in the intermittency cases.

Table 2 Different intermittency method versus kI; Percentages denote $x / c$ for case \#2, and the positions on the plate (case \#1) are expressed as $R e_{x} \times 10^{-4}$

\begin{tabular}{lllllll}
\hline case & Model & $P_{k}^{\lim }$ & Solver & Transition & TargetTrip & ForcedLaminar \\
\hline$\# 2$ & 2-eq. & off & OF-LM & $47-52 \%$ & - & - \\
$\# 2$ & 0 -eq & off & CFX & $17-25 \%$ & $10 \%$ & - \\
$\# 2$ & 0 -eq & off/on & OF-IM & $20-30 \%$ & $10-10.2 \%$ & $0-9.8 \%$ \\
$\# 2$ & $k \mathrm{I}$ & - & OF & $10-11 \%$ & $10-10.2 \%$ & $0-.8 \%$ \\
$\# 1$ & 0 -eq & off/on & OF-IM & $2.3-8.9$ & $0.4-0.6$ & $0-0.3$ \\
$\# 1$ & 0 -eq & off/on & OF-IM & $8.6-18.3$ & $6.7-6.9$ & $0-6.6$ \\
$\# 1$ & $k \mathrm{I}$ & - & OF & $6.7-9.7$ & $6.7-6.9$ & $0-6.6$ \\
\hline
\end{tabular}


It should be noted that the spatial definition of intermittency ( $\gamma$ distribution) may involve the free-stream region as well as the mentioned $x$ range, see Fig. 2. Basically, any modification due to tripping/transition relates solely to the BL and not the flow outside. Therefore, both the injection and laminar regions should be of the size of the BL thickness. However, sensitivity analysis on the size of the laminar region showed that in low-turbulence freestream flows, it can be specified as a larger box, without affecting the results. Indeed, in the test cases of this study, which correspond the models in MTL, the $k$ content of the freestream is inherently quite low. There exists more sensitivity about the injection region size, which will be addressed in Sect. 5 .

The modeled forced transition over the airfoil is accomplished in $\Delta x / c=1 \%$ with the $k$ I method, while it takes $8-10 \%$ with the intermittency models. Over the flat plate, the $k \mathrm{I}$ method results in a transition within $\Delta R e_{x} / R e_{L}=0.9 \%$, while the development length is $\approx 3 \%$ with intermittency models. While the transition is immediate in $k \mathrm{I}$ method, it is observed that with intermittency models, transition initiates with a distance gap from the specified target trip: $\Delta R e_{x} / R e_{L}=0.5 \%$ in flat plate and $\Delta x / c=7-10 \%$ for the airfoil. Using $\gamma-R e_{\theta}$ for case \#2, the flow became FT only at $x / c=0.5$, because the laminar region extended so that $C_{f}$ decreased to zero and the flow separated just before transition. In Fig. $8 C_{f}$ is plotted for the flat plate with the two scenarios of tripping (i.e. early and delayed) for both the $I M$ and the $k I$ methods. It illustrates the fact that even the extra term, $P_{k}^{\lim }$, is not sufficient to produce an immediate transition. For the same target tripping location, the $I M$ requires a longer distance to produce a turbulent flow than the $k \mathrm{I}$ approach, which confirms that the latter is superior when it comes to obtaining rapid transition to turbulence. Since the assessment results were not encouraging for case \#1 and \#2, we did not continue the investigations with case \#3. It is worth noting that all methods lead to the same behavior in the developed turbulent region further downstream, albeit at different BL thicknesses.

\section{Formulating the Modified Injection Method $(\gamma-k \mathrm{l})$}

In the previous section we observed the deficiency of the $I M$ method as a tripping technique, while it has the advantage of being based on a explicit additional term in the equations. The $k \mathrm{I}$ method in Sect. 3 provided the intended tripping but it is based on potentially unclear model parameters. Looking at Eq. (2), $P_{k}^{\text {lim }}$ functions as an extra source of turbulence, which is locally injected and then turns off once the flow is FT. Indeed, the contribution of $P_{k}{ }^{\lim }$ in the $I M$ method can be seen as the common concept with the source term of $S_{k}$ in $k$ I. $P_{k}{ }^{\lim }$ was introduced in the Menter's transition model (Menter et al. 2015), in order to boost the transition where the free stream turbulence is too low to make the transition; and the injected $k$ in $k \mathrm{I}$ method was applied as the supplementary turbulence to compensate the fact that the gradual transition process is skipped. Therefore, $P_{k}^{\lim }$ may be modified to work as a tripping exciter term $S_{k}$. On the other hand, the injection method can be enhanced by avoiding the iterative process to define the involved parameters. Thus, here we propose a general method by combining both approaches, and develop a new formula that may function as a novel tripping model. So, the goal is to reproduce the results in Sect. 3, in a formulated way through modifying the definition of $P_{k} \lim$.

The boost term, $P_{k}^{\lim }$, in Eq. (5) correlates with $S, \Omega$, and $R e_{v}$, which is in accordance to the gradual transition process. These parameters vary rapidly along the flow direction, as well as the perpendicular to the flow. However, the injection method results show that 


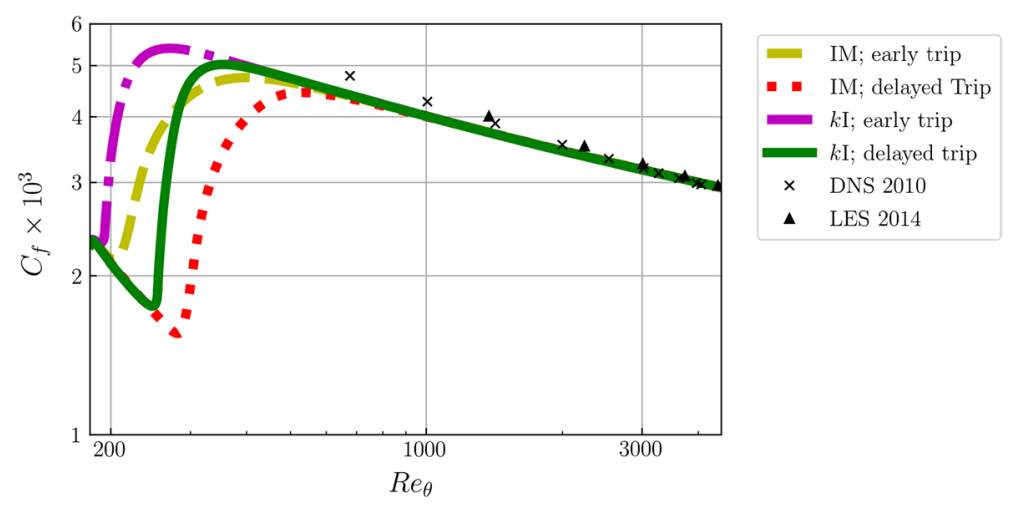

Fig. 8 Intermittency method $(I M)$ versus $k$ injection approach $(k \mathrm{I})$ compared in a flat-plate ZPG TBL. Two tripping scenarios are conducted: early and delayed; FT baselines are based on Eitel-Amor et al. (2014) (LES 2014) and Schlatter and Örlü (2010) (DNS 2010)

the tripping takes place with a constant injection rate and does not correlate with the local properties. Accordingly, the following equations are proposed for the transport of $k$ in a coupled tripping-RANS turbulence model,

$$
\begin{gathered}
\frac{\partial(\rho k)}{\partial t}+\frac{\partial\left(\rho u_{j} k\right)}{\partial x_{j}}=\tilde{P}_{k}+\tilde{P}_{k}^{\lim }-\tilde{D}_{k}+\frac{\partial\left[\left(\mu+\sigma_{k} \mu_{t}\right) \frac{\partial k}{\partial x_{j}}\right]}{\partial x_{j}}, \\
\tilde{P}_{k}^{\lim }=\frac{k_{1}}{A_{\text {inj }}} \max (\gamma-0.2,0)(1-\gamma) \max \left[\frac{3 \mu-\mu_{t}}{\left|3 \mu-\mu_{t}\right|}, 0\right],
\end{gathered}
$$

where the difference between Eqs. 6 and 2 is the use of $\tilde{P}_{k}{ }^{\lim }$ in the former to convert the coupled transition into a coupled-tripping model. $A_{\text {inj }}$ is the area of the specified injected area, and $k_{1}$ is an injection coefficient, introduced as a production/source term over the unit area: $k_{1}=5 / 9 \mathrm{~W} / \mathrm{m}$. The coefficient $\mathrm{k} 1$ is selected similar to the approach in experimental tripping, which is the urpose of the work. The dimension of $\mathrm{k} 1$ shows that it is the required energy to generate the turbulence per the unit span-length. This is similarly obtained by trial and error in the laboratory to find e.g. the proper strength and location of a tripping device.

The formulation given by Eqs. 6 and 7 was implemented in OF as a new library for a modified-SST turbulence model. Without including any other transport equations (0-equation model), the transport equation of $k$ was modified, and we also included the additional changes related to the blending function in the $\omega$ equation, see Sect. 4 for the details. The injection region is specified through a prescribed intermittency value (0.9) for $\gamma$ which is introduced as a field, while in the target laminar and turbulent regions $\gamma$ is set to zero and one respectively. Similar to Fig. 2, the laminar region in the airfoil plane is set to be a box of the size $0.8 c \times 0.3 c$, where the injection region is approximately $0.006 c \times 0.006 c$. As mentioned in previous sections, the laminar box should be confined to the boundary layer if free-stream turbulence is not negligible. However, the size of the laminar box does not affect the WT simulations, in which the turbulence intensity is kept low for the free-stream flow. 
The $3 \mathrm{D}$ domain in the WT was modeled with the wing at angle of attack $8^{\circ}$. With the application of our adapted model, the flow was tripped at the target location. Fig. 9 shows the test section outlines and the mid-height section, contoured with the velocity distribution. A box around the leading edge, including the injection location, is enlarged. The right and left panels show the $\gamma$ value, which is set according to the previous paragraph, and the resulting contours of the turbulent kinetic energy. The $k$ contour, as the measure of turbulence, indicates that the intended sudden jump is achieved at the target location of tripping via $\gamma-k$ I method.

All the other plots in Sects. 3.1, 3.2 and 3.3 ( $k$ I method) are obtained similarly with the application of the $\gamma-k \mathrm{I}$ in OF, i.e. transition is implemented at the target trip location and within a short length. Additionally, there is no need to ad-hoc determine the magnitude of the injection as required for the $k \mathrm{I}$ method. As an example, the chordwise distribution of $C_{f}$ is shown in Fig. 10 for an airfoil at $11^{\circ}$ and $5^{\circ}$ angle of attacks, in which tripping is implemented via both methods of $k \mathrm{I}$ and $\gamma-k \mathrm{I}$. LES data for the tripped airfoil at freeflight condition is also included in Fig. 10b as the reference for $5^{\circ}$ angle of attack. The similarity indicates the same function of $\gamma-k \mathrm{I}$ with a general formulation, compared to the $k$ I method. The two lines for both RANS methods closely follow each other, indicating that the physical effect of both trippings are very similar, and thus the reaction of the BL. Fig. 10a shows tripping at a near-stall angle of attack. A premature transition occurs at $x / c \approx 0.05$ with the standard $k-\omega \mathrm{SST}$, due to the FT assumption throughout the BL. With the application of the $\gamma-k$ I method, the transition is delayed up to the considered tripping point, which however leads to a small laminar separation bubble, indicated by an area with low $C_{f}$. A similar behaviour was obtained using the $k$ I method. Both approaches cause the flow to reattach immediately downstream of the tripping, and all three cases follow a similar behaviour up to the TE. This clearly shows the advantage of the new method, allowing

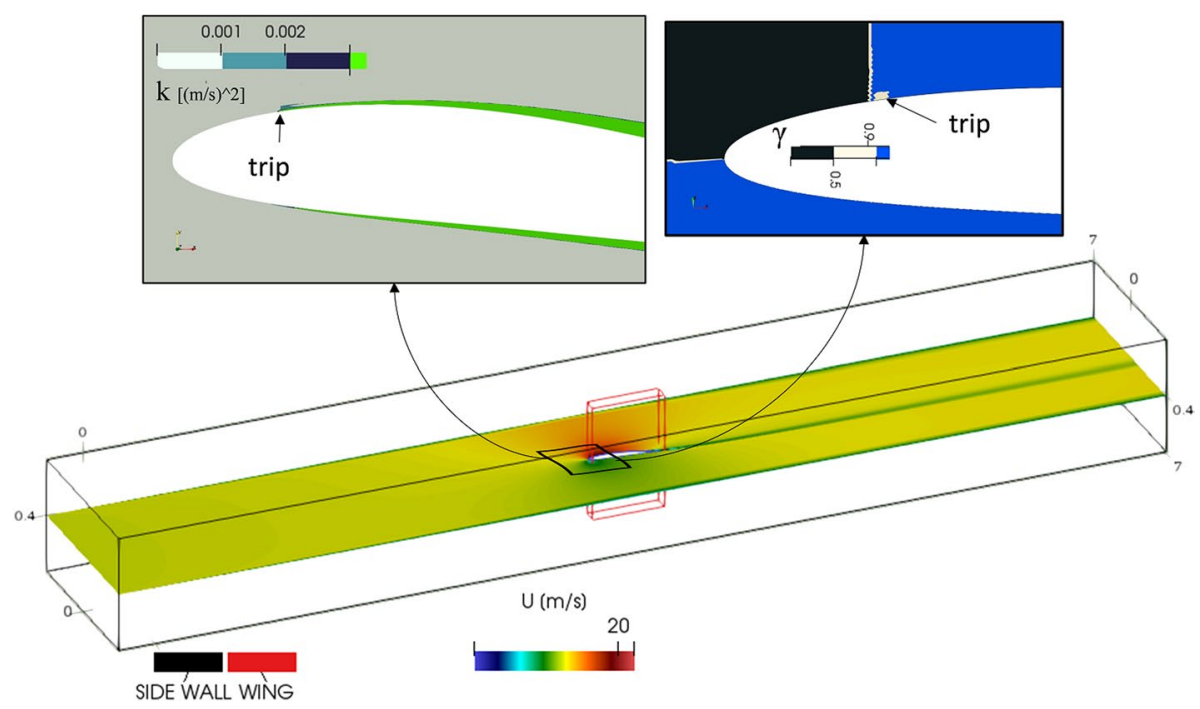

Fig. 9 Tripping in a WT with a wing at $8^{\circ}$ angle of attack. The lower part shows the velocity contours at mid-height section with a specified box around the wing. (The red and black cubes represents the wing location, and the WT side walls respectively.) Right panel: $\gamma$-contour at the selected box, Left panel: $k$-contour in the selected black box 


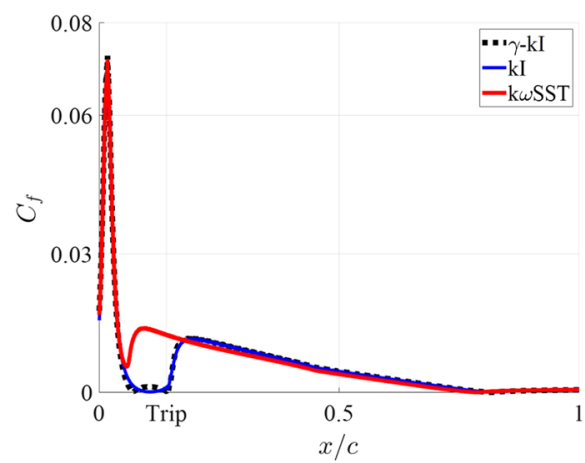

(a) $\mathrm{AOA}=11^{\circ}$

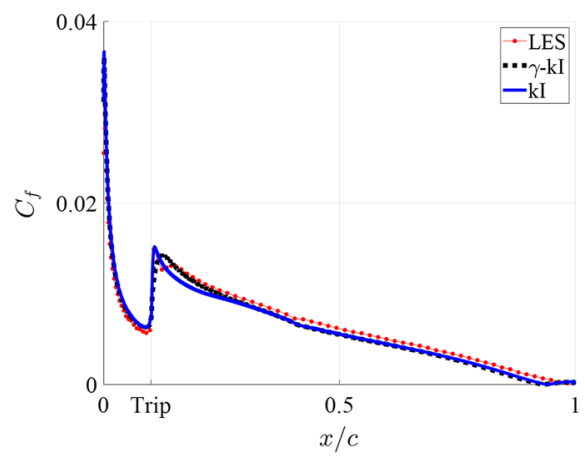

(b) $\mathrm{AOA}=5^{\circ}$

Fig. 10 Chordwise distribution of skin-friction coefficient, $C_{f}$ over the airfoil suction side: $k \mathrm{I}$ vs $\gamma-k \mathrm{I}$; LES data refers to the the same airfoil at free-flight condition. $k-\omega$ SST denotes the standard model formulation (non-tripped)

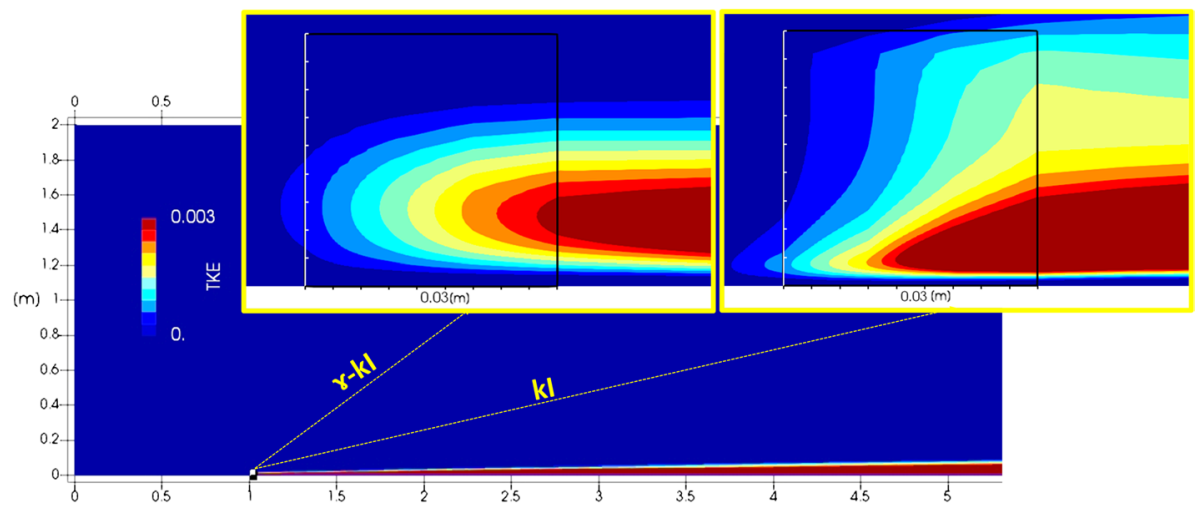

Fig. 11 Tripping on a $7 m$ flat plate at $x=1 m: k$ contour is shown inside the predefined injection area $\left(3 \times 3 \mathrm{~cm}^{2}\right)$ for two approaches of $\gamma-k \mathrm{I}$ and $k \mathrm{I}$ on left and right panels, respectively

to be able to specify a tripping location, without the need for parameter adjustments even at a near stall angle of attack.

The main advantage of the proposed formulation compared to that of the $k \mathrm{I}$ method is the last term in Eq. (7), i.e. $\max \left(\frac{3 \mu-\mu_{t}}{\left|3 \mu-\mu_{t}\right|}, 0\right)$. According to the formulation both in Eqs. (5) and (7), once $\gamma=1$ the boost term becomes inactive. Additionally another term is defined to turn it off even within the injection length, where $\gamma<1$. This is a switch to turn the injection off once the flow is FT but $\gamma$ has not reached one, i.e. the injection area in the $\gamma-k \mathrm{I}$ method. The condition is selected based on Menter's model, in which $\mu_{t}>3 \mu$ is defined as the criterion (Menter et al. 2015). In this way, any unnecessary additional forcing is avoided. Basically the injection zone should mimic the function of a tripping device, e.g. sandpaper. To ensure avoiding any side effects of the injection, this area should be as small as possible. With the switch-off term, we make sure that the injection takes place only at the tripping location and the injection does not change the rest of domain, since it stops once the flow has become turbulent. By means of this switch, the uncertainty in defining the injected area is effectively resolved. In this way the switch term controls where 
the $k$ injection is necessary and excludes the extra cells, which may be included in the specified injection zone, see Fig. 2. Observations from the flow field show that the $\mu_{t}$ increase is not monotonic normal to the wall, however the $\mu_{t}>3 \mu$ criterion can filter the transitioned (FT) cells and cancel the $k$ injection at the parts in which $\mu_{t}$ has already increased.

Figure 11 shows the turbulence injection over the flat plate via $k$ contours. The injection area box is shown for the two methods of $k \mathrm{I}$ and $\gamma-k \mathrm{I}$ in two separate panels (left and right respectively). Although the pre-specified area is the same $\left(3 \times 3 \mathrm{~cm}^{2}\right)$, the injection has been limited in $\gamma-k \mathrm{I}$ and the unnecessary parts are excluded with the application of the switch-off term. Half of the considered box is un-injected at $\gamma-k \mathrm{I}$ method (Fig. 11-left panel), where $k$ is zero on the half top part. This is implemented by the additional term which stops the injection once the transition is fulfilled. With the $k \mathrm{I}$ method (Fig. 11-right panel), the whole predefined injection depth $(3 \mathrm{~cm})$ is affected by the turbulence injection, in which there is no controlling term.

\section{Conclusions and outlook}

The implementation of laminar-turbulent tripping is assessed in a RANS turbulence model, with the aim of replicating the tripping in wind-tunnel models and ultimately developing more reliable aerodynamic simulations, in which the uncertain (and ultimately unnecessary) modeling of the transition process is avoided. Two main features are intended via the numerical tripping in the $k-\omega \mathrm{SST}$ model, which are according to the function of the experimental trip devices: first, the transition onset at the exact target trip location; and second a short development length. These two features provide a controllable transition that ultimately can affect the global flow characteristics. The local changes in the transition behaviour affect the drag, while the laminar-turbulent transition can affect the global flow characteristics by affecting flow separation. Both aspects are thoroughly discussed in the present manuscript.

The results from the turbulence-injection $(k \mathrm{I})$ method show a fair agreement with DNS and LES tripping approaches and experimental data. However, this method relies on two uncertain parameters: the injection area and the magnitude of the required $k$ injection. Thus, this approach requires a calibration process for each test case. We also tested the capabilities of general transition models for tripping even though they were not designed for that purpose: A predefined transition location, applied as $I M$ method, did not result in a desired rapid and controlled transition and therefore these methods are not suitable for tripping. The failure of such models to function as a tripping model show the necessity of a modified formulation in order to match this model with the $k \mathrm{I}$ method.

With the modification in the transition model formulation, both approaches (the $k \mathrm{I}$ and the $I M)$ are combined and the deficiencies from both methods are addressed so that the two aforementioned goals are fulfilled. The newly developed model, $\gamma-k \mathrm{I}$, was tested in a number of flow cases, and the proposed injection method shows advantages in all cases. In particular, the non-physical premature transition, caused by the FT assumption in the formulation of standard RANS, is inhibited and one can control the laminar state and even prolong the laminar state by inhibiting the occurrence of transition.

These results confirm the advantages of the new method compared to the $I M$ method. In comparison with $k \mathrm{I}$ method, the proposed method is not dependent on an ad-hoc process to find the model parameters (i.e. the injection area and magnitude). Therefore the main 
advantage relies on the fact that the uncertain parameters in the previous method can be defined directly via the proposed formula.

The reference turbulence model is $k-\omega$ SST and the base transition model is the $\gamma$ model of Menter et al. (2015). The test cases include a ZPG TBL developing on a flat plate, in addition to the flow around a NACA4412 wing section in free-flight conditions, as well as in 2D and 3D wind-tunnel test sections. Most cases of technical relevance can be simplified, with certain assumptions, to cases similar to the ones discussed in this study. The present work provides evidence for the usefulness and generality of the proposed tripping approach. The behaviour of the friction coefficient $C_{f}$ was adopted as the indicator of the near-wall turbulence region for both ZPG flat plate and airfoils. The ZPG results confirm that the BL adaptation is quicker with tripping at very low $R e_{\theta}$. Compared to the DNS tripping techniques, the achieved RANS tripping results were in agreement with the boundarylayer data.

The tripping technique applied to 3D RANS simulation of a full wind tunnel improves the results significantly so that close agreement between experimental data and the 3D RANS is achieved. Therefore, the proposed tripping method may serve as a potential method to replicate the measured data in a full-scale wind tunnel, as such experiments are typically tripped. This can serve as a general approach to many engineering development studies which are based on tripped cases, and improve the accuracy of the flow predictions, specifically for separated flows.

Acknowledgements The authors are grateful for all the data made available by the other researchers referenced in the text.

Funding Open access funding provided by Royal Institute of Technology. Financial support provided by the Knut and Alice Wallenberg Foundation is gratefully acknowledged. The computations were enabled by resources provided by the Swedish National Infrastructure for Computing (SNIC) at PDC and HPC2N partially funded by the Swedish Research Council through grant agreement no. 2020/3-5.

\section{Declarations}

Conflict of interest The authors declare that they have no conflict of interest.

Open Access This article is licensed under a Creative Commons Attribution 4.0 International License, which permits use, sharing, adaptation, distribution and reproduction in any medium or format, as long as you give appropriate credit to the original author(s) and the source, provide a link to the Creative Commons licence, and indicate if changes were made. The images or other third party material in this article are included in the article's Creative Commons licence, unless indicated otherwise in a credit line to the material. If material is not included in the article's Creative Commons licence and your intended use is not permitted by statutory regulation or exceeds the permitted use, you will need to obtain permission directly from the copyright holder. To view a copy of this licence, visit http://creativecommons.org/licenses/by/4.0/.

\section{References}

Acarer, S.: Critical study of the effects and numerical simulations of boundary layer transition in lift-based wind turbines at moderate reynolds numbers. J. Renew. Sustain. Energy 12(6), 063309 (2020).

ANSYS, Inc.: ICEM CFD User Manual (2011). Release 14.0

ANSYS, Inc.: ANSYS CFX-Solver Theory Guide (2015). Release 16.2

Blackwell, J.A.: Preliminary study of effects of reynolds number and boundary-layer transition location on shock-induced separation Tech. rep. NASA Langley Research Center Hampton, VA, United States (1969) 
Bross M., Scharnowski S., Kähler C.: Influence of leading edge tripping devices on supersonic turbulent boundary layer characteristics. In: The 5th International Conference on Experimental Fluid Mechanics, ICEFM 2018 Munich (2018)

Coles, D., Wadcock, A.J.: Flying-hot-wire study of flow past an NACA 4412 airfoil at maximum lift. AIAA J. 17(4), 321-329 (1979)

dos Santos, F.L., Sanders, M.P., de Santana, L.D., Venner, C.H.: Influence of tripping devices in hastening transition in a flat plate submitted to zero and favorable pressure gradients. In: AIAA 2020-0046. AIAA Scitech 2020 Forum (2020)

Eitel-Amor, G., Örlü, R., Schlatter, P.: Simulation and validation of a spatially evolving turbulent boundary layer up to Re=8300. Int. J. Heat Fluid Flow 47, 57-69 (2014)

Erm, L.P., Joubert, P.N.: Low-Reynolds-number turbulent boundary layers. J. Fluid Mech. 230, 1-44 (1991)

Fahland, G.: Flow control for turbulent skin-friction drag reduction on airfoils. Master's thesis, Karlsruhe Institute of Technology (2019)

Gerolymos, G., Vallet, I.: Bypass transition and tripping in Reynolds-stress model computations. In: AIAA 2013-2425. 21st AIAA Computational Fluid Dynamics Conference (2013)

Geuzaine, C., Remacle, J.F.: Gmsh: A 3-d finite element mesh generator with built-in pre- and post-processing facilities. Int. J. Numer. Methods Eng. 79(11), 1309-1331 (2009)

Gokdepe, M.: Turbulence models for the numerical prediction of transitional flows with ranse. Ph.D. thesis, Department of Mechanical Engineering (2015)

Greenshields, C.J.: OpenFOAM User Guide (2019). Version 7

Head, M.R., Bandyopadhyay, P.: New aspects of turbulent boundary-layer structure. J. Fluid Mech. 107, 297-338 (1981)

Iverson, D., Boudreau, M., Dumas, G., Oshkai, P.: Boundary layer tripping on moderate reynolds number oscillating foils. J. Fluids Struct. 86, 1-12 (2019)

Langtry, R.: A correlation-based transition model using local variables for unstructured parallelized cfd codes. Ph.D. thesis, University of Stuttgart (2006)

Lewis, J.B.: Effect of boundary-layer tripping on turbulence generation and trailing-edge noise in transitional airfoils. Master's thesis, Embry-Riddle Aeronautical University (2017)

Lindgren, B., Johansson, A.V.: Evaluation of the flow quality in the MTL wind-tunnel. Tech. Rep. TRITAMEK 2002:13, Dept. of Mechanics, KTH, Stockholm (2002)

Luckring, J., Deere, K., Childs, R., Stremel, P., Long, K.: An application of CFD to guide forced boundarylayer transition for low-speed tests of a hybrid wing-body configuration. In: 32nd AIAA Aerodynamic Measurement Technology and Ground Testing Conference (2016)

Mabey, D.: A review of scale effects in unsteady aerodynamics. Progress Aerospace Sci. 28(4), 273-321 (1991)

Mallor, F.: Enabling high-fidelity measurements of turbulent boundary layer flow over wing sections in the MTL wind tunnel. Master's thesis, KTH, Mechanics (2019)

Mallor, F., Dogan, E., Atzori, M., Parikh, A., Vinuesa, R., Örlü, R., Schlatter, P.: Design considerations for the flow conditions around a wing model inside a wind tunnel. In: Svenska Mekanikdagar, KTH. Svenska Mekanikdagar, KTH (2019)

Marusic, I., Chauhan, K., Kulandaivelu, V., Hutchins, N.: Evolution of zero-pressure-gradient boundary layers from different tripping conditions. J. Fluid Mech. 783, 379-411 (2015)

Menter, F., Smirnov, P., Liu, T., Avancha, R.: A one-equation local correlation-based transition model. Flow Turbul. Combust. 95, 1-37 (2015)

Menter, F.R.: Two-equation eddy-viscosity turbulence models for engineering applications. AIAA J. 32(8), 1598-1605 (1994)

Monkewitz, P.A., Chauhan, K.A., Nagib, H.M.: Self-consistent high-Reynolds-number asymptotics for zero-pressure-gradient turbulent boundary layers. Phys. Fluids 19(11), 115101 (2007)

Österlund, J.M.: Experimental studies of zero pressure-gradient turbulent boundary layer flow. Ph.D. thesis, KTH, Mechanics (1999). NR 20140805

Peterson, J.B.: Boundary-layer velocity profiles downstream of three-dimensional transition trips on a flat plate at Mach 3 and 4 Tech. rep. NASA Langley Research Center Hampton, VA, United States (1969)

Rengasamy, K., Mandal, A.C.: Experiments on effective tripping device in a zero pressure gradient turbulent boundary layer. J. Phys. Conf. Series 822, 012016 (2017)

Reshotko, E.: Transient growth: a factor in bypass transition. Phys. Fluids 13(5), 1067-1075 (2001)

Roy, C., Blottner, F.: Assessment of one- and two-equation turbulence models for hypersonic transitional flows. J. Spacecraft Rockets 38, 699-710 (2001)

Rumsey, C.: Turbulence modeling resource (2021). Langley Research Center

Sanmiguel Vila, C., Vinuesa, R., Discetti, S., Ianiro, A., Schlatter, P., Örlü, R.: On the identification of wellbehaved turbulent boundary layers. J. Fluid Mech. 822, 109-138 (2017) 
Schlatter, P., Örlü, R.: Assessment of direct numerical simulation data of turbulent boundary layers. J. Fluid Mech. 659, 116-126 (2010)

Schlatter, P., Örlü, R., Li, Q., Brethouwer, G., Fransson, J.H.M., Johansson, A.V., Alfredsson, P.H., Henningson, D.S.: Turbulent boundary layers up to $\operatorname{Re}_{\Theta}=2500$ studied through simulation and experiment. Phys. Fluids 21(5), 051702 (2009)

Schlatter, P., Örlü, R.: Turbulent boundary layers at moderate Reynolds numbers: inflow length and tripping effects. J. Fluid Mech. 710, 5-34 (2012)

Spalart, P., Allmaras, S.: A one-equation turbulence model for aerodynamic flows. In: 30th Aerospace Sciences Meeting and Exhibit (1992)

Sreejith, B., Sathyabhama, A.: Numerical study on effect of boundary layer trips on aerodynamic performance of e216 airfoil. Eng. Sci. Technol. Int. J. 21(1), 77-88 (2018)

Tabatabaei, N.: Impact of icing on wind turbines aerodynamic. Ph.D. thesis, Luleå University of Technology, Fluid and Experimental Mechanics (2018)

Tabatabaei, N., Örlü, R., Vinuesa, R., Schlatter, P.: Aerodynamic free-flight conditions in wind tunnel modelling through reduced-order wall inserts. Fluids 6(8), 265 (2021)

Vinuesa, R., Negi, P., Atzori, M., Hanifi, A., Henningson, D., Schlatter, P.: Turbulent boundary layers around wing sections up to $R e_{c}=1,000,000$. Int. J. Heat Fluid Flow 72, 86-99 (2018)

Walters, D.K., Cokljat, D.: A three-equation eddy-viscosity model for reynolds-averaged navier-stokes simulations of transitional flow. J. Fluids Eng.-Trans. ASME 130, 121401 (2008) 